\title{
Amphibian Toxicology: A Rich But Underappreciated Model for Ecotoxicology Research
}

\author{
Valérie S. Langlois ${ }^{1}$ \\ Accepted: 4 April 2021 / Published online: 11 April 2021 \\ (c) The Author(s), under exclusive licence to Springer Science+Business Media, LLC, part of Springer Nature 2021
}

\section{Background}

Thanks to the high degree of synteny with the human genome, the Western clawed frog has emerged in the last decade as a superior non-mammalian vertebrate model for studying genes that are related to human diseases (Dimitrakopoulou et al. 2019; Nenni et al. 2019). Amphibian species have also been recognized as sensitive laboratory models for extrapolating the effects of exposure to endocrine disrupting chemicals (EDCs) to humans (Holbech et al. 2020). However, when entering the keywords "toxicity" and either "fish", "amphibian", "reptile", "bird", or "mammalian" in PubMed (accessed on March 1st, 2021), studies with amphibians encompass approximatively $10 \%$ of the published primary literature when excluding mammalian species, and below $0.6 \%$ when including mammals (Fig. 1). A "species discrimination" exists even in ecotoxicological research where funding to support the field of amphibian toxicology remains scarce.

In terms of evolution, ecology, and physiology, amphibians represent the transition between aquatic and terrestrial environments, and are therefore, a valuable laboratory model when testing for contaminants that are released into both aquatic and terrestrial ecosystems (Johnson et al. 2017). The extraordinary changes in morphology, physiology, endocrine balance, cellular differentiation, and gene expression that occur in amphibians during metamorphosis make these species especially sensitive to the effects of contaminants, and thus, amphibians are highly valuable vertebrate models for laboratory testing or as bioindicators of contamination in the field. This is especially the case for detecting responses to EDCs that could interfere with this crucial life event (Carr and Patiño 2012). There is evidence that population-level

Valérie S. Langlois

valerie.langlois@inrs.ca

1 Centre Eau Terre Environnement, Institut National de La Recherche Scientifique (INRS), Québec, QC, Canada responses to toxic chemicals may be a factor contributing to global declines in amphibians (Egea-Serrano et al. 2012).

Significant advances in genomics have increased access for the scientific community to the genomes of amphibians. For instance, the genome of the American bullfrog, was recently published and has already shown its sensitivity to thyroid hormone challenge (Hammond et al. 2017), highlighting its promising utility in the field of ecotoxicology. With an increasing emphasis on "reducing, replacing, and refining" testing with vertebrates (also known as the $3 \mathrm{Rs}$ ), the advantages for in vivo testing using some amphibian species include the large clutch sizes that yield early life stages for conducting embryotoxicity assays, thereby reducing reliance on testing with large numbers of sentient adults. In addition, recent advances in cell line development and reporter gene assays provide new tools for in vitro testing that can contribute to ecotoxicological assessments (Scholz et al. 2013; Houck et al. 2021).

\section{Special Issue Articles}

Within this context, this special issue of AECT presents a collection of 12 articles describing recent work conducted in the field of amphibian toxicology. A diverse range of toxic agents were evaluated in these studies, including road salt, metals, several chemical pesticides, biopesticides, a synthetic progestin, an artificial sweetener (sucralose), and an ingredient in some pharmaceuticals (dioctyl sodium sulfosuccinate). The toxicological endpoints evaluated in response to exposure to these agents included mortality, morphometric changes, developmental effects, gonadal malformation, thyroid gland development, hormone levels, neuromast development, gene expression, and behavior. The amphibian species studied either with in vivo laboratory protocols or by field monitoring included Ambystoma gracile (northwestern salamander), Lithobates pipiens (northern leopard frog), Lithobates catesbeianus (American bullfrog), Lithobates 
Fig. 1 Compilation of papers published since 1900 on toxicity for each of the vertebrate classes. The keywords "toxicity" and either "fish", "amphibian", "reptile", "bird", or "mammalian" were used to generate the search in PubMed (accessed on March 1st, 2021)

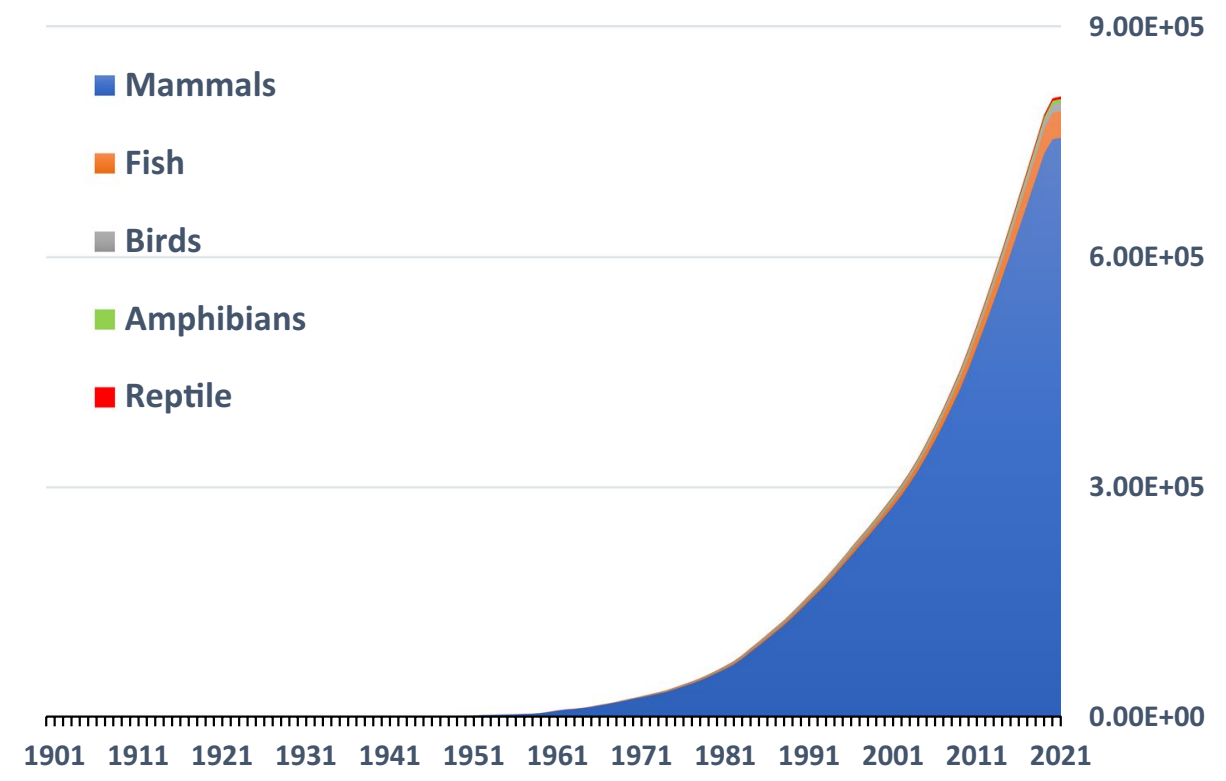

sylvaticus (wood frog), Rhinella arenarum (Argentine toad), Silurana tropicalis (Western clawed frog), and Xenopus laevis (African clawed frog). This research was conducted by colleagues based in Nigeria, Argentina, Canada, and the United States of America.

From this standpoint, the articles included in this special issue will provide insights into the effects of exposure to environmentally relevant concentrations of contaminants on the health of amphibians and will illustrate the breadth of important information that this vertebrate model can yield.

Acknowledgements We thank all the colleagues who contributed and peer-reviewed the articles that are included in this special issue.

\section{References}

Carr JA, Patiño R (2012) The hypothalamus-pituitary-thyroid axis in teleosts and amphibians: endocrine disruption and its consequences to natural populations. Gen Comp Endocrinol 170:299-312

Dimitrakopoulou D, Tulkens D, Vlierberghe P, Vleminck K (2019) Xenopus tropicalis: joining the armada in the fight against blood cancer. Front Physiol 1(10):48
Egea-Serrano A, Relyea RA, Tejedo M, Torralva M (2012) Understanding of the impact of chemicals on amphibians: a meta-analytic review. Ecol Evol 2:1382-1397

Hammond S, Warren RL, Vandervalk BP et al (2017) The North American bullfrog draft genome provides insight into hormonal regulation of long noncoding RNA. Nat Commun 8:1433

Holbech H, Matthiessen P, Hansen M et al (2020) ERGO: breaking down the wall between human health and environmental testing of endocrine disrupters. Int J Mol Sci 21:2954. https://doi.org/10. 3390/ijms21082954

Houck KA, Simha A, Bone A et al. (2021) Evaluation of a multiplexed, multispecies nuclear receptor assay for chemical hazard assessment. Toxicology In Vitro, Apr; 72:105016. Open access: Doi: https://doi.org/10.1016/j.tiv.2020.105016. Epub 2020 Oct 10

Johnson MS, Aubee C, Salice CJ et al (2017) A review of ecological risk assessment methods for amphibians: comparative assessment of testing methodologies and available data. Integr Environ Assess Manag 13:601-613

Nenni MJ, Fisher ME, James-Zorn C et al (2019) Xenbase: facilitating the use of Xenopus to model human disease. Front Physiol 10:154. https://doi.org/10.3389/fphys.2019.00154

Scholz S, Renner P, Belanger SE et al (2013) Alternatives to in vivo tests to detect endocrine disrupting chemicals (EDCs) in fish and amphibians-screening for estrogen, androgen and thyroid hormone disruptions. Crit Rev Toxicol 43:45-73 